\title{
EI desarrollo de la autonomía mediante las técnicas de aprendizaje cooperativo en el aula de 12
}

\author{
IRENE SÁNCHEZ GodOY \\ Sonia Casal Madinabeitia \\ Universidad Pablo de Olavide
}

Recivido: 9 julio 2014 / Aceptado: 19 marzo 2015

ISSN: $1697-7467$

\begin{abstract}
RESUMEN: El Aprendizaje Cooperativo, enfoque de trabajo en grupos estructurados utilizado en todos los niveles educativos, concibe el proceso de enseñanza/aprendizaje como un fenómeno eminentemente social. La autonomía, por otro lado, es la capacidad de controlar el propio aprendizaje y, por lo tanto, un proceso individual.

Este artículo persigue reforzar los vínculos que unen las técnicas de Aprendizaje Cooperativo y la autonomía, alegando que aquellas pueden llegar a desarrollar esta última gracias a los siguientes factores presentes en su puesta en marcha: la reducción de la ansiedad, el aumento de la motivación y la atención a los estilos de aprendizaje.

Palabras Clave: Aprendizaje Cooperativo, autonomía, motivación, ansiedad, estilos de aprendizaje.
\end{abstract}

Promoting Learner Autonomy Through Cooperative Learning Techniques in the L2 Classroom

\begin{abstract}
Cooperative Learning, the pedagogic use of structured group work in educational settings (from kindergarten to university) highlights the learning/teaching process as a social endeavour. Autonomy, on the other hand, is the ability to control one's own learning and is perceived, therefore, as an individual process.

This article aims at corroborating the links between Cooperative Learning techniques and autonomy, since the former can help develop the latter thanks to the conditions included in its implementation and analysed throughout the article: anxiety reduction, increase of motivation and the explicit attention to learning styles.
\end{abstract}

Keywords: Cooperative Learning, autonomy, motivation, anxiety, learning styles.

\section{INTRODUCCIÓN}

Holec (1981, citado en Zoghi y Nezhad, 2012:22) define la autonomía como la habilidad de hacerse cargo del aprendizaje de uno mismo, y añade que esta habilidad no es innata sino que debe ser adquirida, bien por medios naturales, o bien a través de la educación formal. Aoki (2000) hace referencia a tres componentes de la autonomía en este contexto formal. Por un lado, el estudiante debe elegir qué, cómo y por qué aprender. En segundo lugar, debe llevar a cabo un plan y, por último, debe evaluar el resultado de su aprendizaje.

Este último componente de la autonomía (la autoevaluación) resulta fundamental en el proceso de enseñanza/aprendizaje de cualquier estudiante, puesto que le puede propor- 
cionar información imprescindible con el fin de regular su propio proceso de construcción de significados y llegar al objetivo último de la educación: el autoaprendizaje o aprender a aprender, esto es, la capacidad de utilizar los conocimientos adquiridos para adquirir nuevas comprensiones de manera autónoma e individual (Coll y Martín, 1999:178). Moreno y Martínez (2007:51), afirman que autonomía y autoaprendizaje son objetivos centrales de la educación y autores como Ruiz (1997:185) destacan que autonomía y aprender a aprender van de la mano: "para conseguir que el alumno adquiera autonomía y desarrolle un papel activo desde un punto de vista pedagógico, deberá aprender a aprender."

La autonomía en contextos educativos es mencionada en la recomendación 2066/962/ EC, del Parlamento Europeo y del Consejo de 18 de diciembre de 2006, en la definición de las competencias clave para el aprendizaje para toda la vida, puesto que resulta una pieza básica (ver el enlace de la red de competencias clave en el ámbito educativo: http:// keyconet.eun.org/key-competences). Posteriormente, estas ocho competencias europeas han sido reformuladas en siete en la LOMCE (Real Decreto 126/2014, de 28 de febrero por el que se establece el currículo básico de la educación primaria), sustituyendo a las anteriores ocho competencias básicas. De estas competencias clave, las que nos interesan destacar en el presente artículo por su relación con la autonomía son: 'sentido de la iniciativa y espíritu emprendedor' y 'aprender a aprender'.

El documento Competencias Clave del Aprendizaje Permanente (2007:11) define la competencia del 'sentido de la iniciativa y espíritu empresarial' como "la habilidad de la persona para transformar las ideas en actos. Está relacionado con la creatividad, la innovación y la asunción de riesgos, así como la habilidad para planificar y gestionar proyectos con el fin de alcanzar objetivos" y relaciona dicha competencia con la gestión proactiva de proyectos, con la representación y negociación efectiva así como con la habilidad para trabajar tanto individualmente como de manera colaborativa dentro de un equipo.

En cuanto a la competencia de 'aprender a aprender', este mismo documento destaca la necesidad de adquirir ciertas capacidades básicas como la lectura, la escritura, el cálculo y las Tecnologías de la Sociedad y la Información (TSI) de modo que los aprendices asienten unas bases para acceder a nuevas capacidades y conocimientos. Para ello, explica también:

De las personas se espera que sean autónomas y autodisciplinadas en el aprendizaje, pero también que sean capaces de trabajar en equipo, de sacar partido de su participación en un grupo heterogéneo y de compartir lo que hayan aprendido. Las personas deben ser capaces de organizar su propio proceso de aprendizaje, de evaluar su propio trabajo y, llegado el caso, de procurarse asesoramiento, información y apoyo. (Competencias Clave del Aprendizaje Permanente, 2007:8).

Las citas anteriores hacen referencia al trabajo en equipo ('negociación efectiva', 'sacar partido de su participación en un grupo heterogéneo', 'compartir lo que hayan aprendido'), lo que nos conduce al segundo punto de interés de nuestro artículo: el Aprendizaje Cooperativo, enfoque metodológico caracterizado por el hecho de que los procesos de aprendizaje han de producirse necesariamente de manera social, ya que al menos debe haber dos individuos en interacción, y ha de darse además una relación de interdependencia (Johnson et al., 1994:9).

A pesar de los numerosos estudios realizados en aras de investigar los beneficios y ventajas que se derivan del Aprendizaje Cooperativo, así como los agrupamientos, técnicas 
o materiales posibles que se pueden dar en todos los niveles educativos (Ver Basta, 2011; Casal, 2005; Gillies y Ashman (eds.), 2003; Johnson, 1993; Law, 2008; León, 2002), existen aún algunos aspectos poco explorados. Autores como García (2005:116), por ejemplo, señalan que la autonomía "ha sido con frecuencia vista en oposición al trabajo en grupos, hasta el punto de que ambas son consideradas a menudo como modos opuestos de organizar la enseñanza." En otras palabras, resulta difícil percibir con claridad la relación entre la interacción social que promueve el Aprendizaje Cooperativo y el desarrollo de capacidades más centradas en el individuo como la autonomía y el aprender a aprender.

Sin embargo, existe un elemento fundamental en el Aprendizaje Cooperativo para llegar a la autonomía: la responsabilidad individual. Junto con la responsabilidad individual, los elementos que contribuyen a que el Aprendizaje Cooperativo funcione eficazmente son: la interdependencia positiva, la interacción cara a cara estimuladora, las técnicas interpersonales y de equipo y la evaluación grupal. (Johnson et al., 1994:8-10). Es interesante observar la relación que se establece entre el desarrollo de la responsabilidad individual y su influencia en la implicación y organización de las actividades colectivas. Citando a Johnson et al., (1994:36):

La responsabilidad individual es la clave para garantizar que todos los miembros del grupo se fortalezcan al aprender cooperativamente. El propósito de los grupos cooperativos, después de todo, es hacer de cada alumno un individuo más fuerte. Durante el aprendizaje cooperativo, los alumnos aprenden conocimientos, destrezas, estrategias o procedimientos dentro de un grupo, y luego los aplican por sí solos para demostrar su dominio personal del material aprendido. Los alumnos aprenden juntos cómo desempeñarse aun mejor individualmente.

Se deduce, pues, que del fomento de la responsabilidad individual que requiere el Aprendizaje Cooperativo depende en gran medida la posterior autonomía y autorregulación que conseguirán los estudiantes. (Holec, 1981, citado en Xu, 2012:2). Como afirma García (2005:109): "la autonomía es un objetivo educativo prioritario en todos los niveles [...] que debe plasmarse por medio de la asunción de responsabilidad por parte de los alumnos."

Wang (2011:274) sostiene que, al tomar responsabilidad, los estudiantes deben hacerse cargo de obligaciones que, tradicionalmente, ha desempeñado el profesorado, tales como diseñar los objetivos de aprendizaje, seleccionar la metodología o evaluar el resultado. Esta mayor implicación de los estudiantes supone a su vez una actitud de madurez y asertividad, constancia, interés y esfuerzo encaminados al logro de ciertas metas y objetivos, actitudes que se adquieren y/o potencian a través de las técnicas del Aprendizaje Cooperativo. El docente va liberando a los estudiantes para que vayan tomando una responsabilidad progresiva de su propio aprendizaje. En palabras de Moreno y Martínez (2007:52):

[...] en el ámbito educativo suelen considerarse que son autónomas aquellas tareas del aprendiz consistentes en resolver ejercicios por sí mismos, plantear nuevos problemas, discutir en grupo algún tema, realizar investigaciones y cualquier actividad realizada fuera del horario de clase o sin el concurso del profesor.

Junto con las características mencionadas por Moreno y Martínez (2007), ser un estudiante autónomo en el aula de L2 implica saber cómo usar herramientas (diccionarios, 
libros de referencia, cuaderno de notas o glosarios); corregir errores propios y ajenos; tomar notas; resumir; realizar una presentación oral; evaluar a los compañeros/as y saber trabajar en grupos (Barbero et al., 2001:35).

Autores como Zogui y Nezhad (2012:23) sostienen que es el propio sistema educativo el que obstaculiza el desarrollo de una conducta autónoma de los discentes, en la que el docente dispone y organiza la formación de los estudiantes sin contar con sus intereses e iniciativas (Zogui y Nezhad, 2012:23). Se precisa, por tanto, de educadores que desarrollen un marco ideal en cuanto a la visión de la educación como liberación y empoderamiento, esto es, un cambio en nuestro modelo educativo en el que el profesorado sea transformador más que reproductor respecto a la manera de enseñar y aprender (Vieira et al., 2004:218). En esta misma línea, Ruiz (1997:187-188) resuelve que:

[...] bajo una perspectiva autodirigida, el rol del educador va a ser más completo, y en un principio más complejo que el existente en los métodos tradicionales, ya que va a requerir el conocimiento no sólo lingüístico del idioma en cuestión sino también la posesión de habilidades comunicativas y metodológicas. Así pues, el rol del orientador va a ser mucho más creativo y variado. En ocasiones deberá a aprender él mismo a enseñar, deberá "entrenarse" para que la relación con el alumno se vea fortalecida.

Un segundo factor que obstaculiza el desarrollo de la autonomía en el aula es la ausencia de involucración del alumnado de todos los niveles en su propio proceso de aprendizaje (Balçikanli, 2010:98-99). Picón (2012:157), en su estudio sobre el fomento de la autonomía en el alumnado en educación secundaria, sostiene que una de las técnicas más efectivas para lograrlo es a través del desarrollo del pensamiento crítico y la implicación del alumnado en la evaluación de su aprendizaje. Por tanto, somos los educadores quienes tenemos que transformar la docencia y abrirla a los estudiantes, fomentando una actitud de responsabilidad y de empoderamiento del propio proceso de transformación o aprendizaje. Es responsabilidad de los docentes y las instituciones apoyar la práctica de la autonomía de los discentes (Aoki, 2000).

El presente artículo tiene como objetivo fundamental reforzar el vínculo existente entre las técnicas de Aprendizaje Cooperativo y el desarrollo de la autonomía en el aula de L2. La reducción de la ansiedad del estudiante, mediante la creación de una atmósfera de aprendizaje positiva; la motivación del alumnado gracias a su mayor implicación en las tareas de clase y la atención a los diferentes estilos de aprendizaje son los puntos sobre los que se puede construir la autonomía desde la socialización y que van a ser analizados en los siguientes apartados.

\section{Factores individuales EN El aprendizaje CoOperativo Que INCIDEN EN LA AUTONOMÍA DEL ALUMNADO}

\subsection{Reducción de la ansiedad hacia la L2}

Como han puesto de manifiesto distintas investigaciones (Horwitz, et al., 1986; Rubio, 2004), la ansiedad, entendida como "un sentimiento de tensión y aprensión asociado espe- 
cíficamente a contextos de segunda lengua, que incluyen las destrezas de hablar (speaking), escuchar (listening) y el aprendizaje en sí mismo." (MacIntyre y Gardner, 1994:284) tiene un efecto debilitante en el proceso de aprendizaje de una L2 puesto que actúa negativamente sobre el autoconcepto del estudiante y, por ende, sobre el desarrollo de su autonomía.

El aprendizaje de una L2 supone, en la mayoría de los casos, un desafío para aquellos estudiantes que han venido presentando dificultades en su aprendizaje. Tal y como Canfield y Wells (1994, citados en Arnold y Douglas, 2000:30) señalan:

[...] es probable que el alumno que ha tenido un rendimiento excelente en el pasado se arriesgue a volver a tener éxito, si no lo consigue su autoestima puede "permitírselo". Un alumno en el que predominan los fracasos será reacio a volver a arriesgarse por temor al fracaso. Su auto-concepto reducido no puede permitírselo.

Por este motivo, aquellos discentes que no se encuentren cómodos con la L2, pueden reducir el nivel de ansiedad en los grupos cooperativos al compartir la responsabilidad del aprendizaje y al intercambiar impresiones con sus iguales. La ansiedad que en ocasiones padecen los aprendices de una L2 debe ser considerada por los docentes en tanto que dicha sensación supone un desgaste, dada la intensidad cognitiva que se emplea en el aprendizaje de una L2. (Ahmed y Zafar, 2010:200).

El estudio realizado por Tsiplakides (2009:41) concluye que la falta de participación por parte del alumnado en actividades orales no se debe a que los aprendices no valoren el aprendizaje del inglés, a razones de pereza o a la falta de interés, sino a un bajo autoconcepto en el uso de la L2. Por ello, una de las propuestas que realiza este autor para reducir los niveles de ansiedad y aumentar la implicación y autonomía del alumnado es el trabajo en un proyecto donde el estudiante tenga un rol y una responsabilidad. Asimismo, añade que "crear una comunidad de aprendizaje proporciona un ambiente de motivación óptima" (Alderman, 2004, citado en Tsiplakides, 2009:41) y "una atmósfera de colaboración" (Gregersen, 2003, citado en Tsiplakides, 2009:41).

Autores como Denegri et al., (2007:33) apuntan que el aumento del autoconcepto se produce en las dimensiones físicas, familiares, personales y sociales al trabajar de forma cooperativa regularmente. Por tanto, el docente debe ser un guía en la implementación del Aprendizaje Cooperativo, un facilitador de los aprendizajes a través de tareas motivadoras y de ambientes de cooperación en aras de crear en el alumnado una sensación de seguridad y autocontrol de modo que pueda sentirse relajado e implicado en los procesos de aprendizaje.

A este respecto, García y Cruz (2013:295) afirman que "el rol del profesor puede ser muy importante ya que gestionará todo lo que ocurra en el aula, y así su actuación con respecto a los alumnos extrovertidos e introvertidos será primordial para reducir los niveles de ansiedad.” En esta misma línea, Martínez (2001:261) considera

[...] fundamental crear las condiciones favorables para fomentar el desarrollo de un entorno de aprendizaje óptimo para el aprendizaje. La instrucción formal debe eliminar o bien reducir la influencia de determinados estados de ansiedad, fomentando la aparición de sensaciones de seguridad y confianza.

\footnotetext{
${ }^{1}$ Traducción propia
} 
En el trabajo realizado por Trujillo (2002:158), se indica que "se ha demostrado con multitud de estudios la efectividad del Aprendizaje Cooperativo en relación con los logros académicos y el desarrollo afectivo, cognitivo y social del individuo" y en un estudio posterior de Vera (2009:6) sobre las consecuencias positivas del Aprendizaje Cooperativo, indica que algunas de ellas son el aumento de la autoestima, la responsabilidad compartida y la oportunidad de crear espacios donde superar las dificultades que alguien pueda tener en un ambiente de compañerismo y confianza.

En palabras de Otero (2009:17): "el aprendizaje cooperativo contribuye a reducir la ansiedad en la medida que fomenta la autoestima de los alumnos y la confianza en sí mismos". Por tanto, los estados afectivos y emocionales negativos que pudiera experimentar un estudiante y que actúan en detrimento de su autonomía, como son la ansiedad, la tensión, el estrés o el miedo a la participación pública en el aula de L2, propios de personas con un bajo autoconcepto o personas introvertidas, pueden verse neutralizados gracias al Aprendizaje Cooperativo, donde los círculos de colaboración recrean un clima de participación inevitable pero de apoyo y empatía hacia el resto de sus integrantes. Una atmósfera de aprendizaje adecuada es el primer paso para el desarrollo de la autonomía en el alumnado (Aoki, 2000).

\subsection{Aumento de la Motivación}

La motivación destaca por ser la responsable de fomentar y afianzar en el estudiante ciertos hábitos o conductas; en este caso, la autonomía. Tal y como señalan Saravia y Bernaus (2008:164) "la actitud y la motivación son esenciales porque sin motivación no hay aprendizaje y porque la actitud apoya dicha motivación." Existen numerosos estudios centrados en los tipos de motivación que un sujeto puede experimentar, así como los incentivos o reforzadores encargados de iniciar dichos hábitos. (Deci et al., 1991; Dörnyei, 1994).

En general, al hablar de motivación se establece la dicotomía entre motivación intrínseca (cuando los motivos que conducen al estudiante hacia el aprendizaje son inherentes) y motivación extrínseca (cuando el motivo que impulsa el aprendizaje es ajeno al propio aprendizaje, y viene determinado por incentivos o reforzadores positivos o negativos). Sin embargo, el objeto de estudio que nos proponemos requiere de una mayor especificación a la hora de analizar los tipos de motivación que puede experimentar el alumnado con respecto al aprendizaje de una L2. La psicología cognitiva establece que la motivación intrínseca parece favorecer la autonomía en el aprendizaje, contribuyendo a fomentar un juicio independiente, mientras que la motivación extrínseca desprende una excesiva dependencia del docente a la hora de solucionar los problemas (Martínez, 2001:241). En este sentido, se suma la aportación de Otero (2009:19), cuando especifica que "el aprendizaje cooperativo tiende hacia una motivación intrínseca, basada en la satisfacción y el gozo de incrementar los conocimientos y la competencia propios, de beneficiar a los otros, etc."

Autores como Gardner y Lambert (1972, citados en Martínez, 2001:242), establecieron su propia tipología con respecto a la naturaleza de la motivación en el aprendizaje de una L2, distinguiendo entre la motivación integradora (producida de manera intrínseca) y la motivación instrumental (engendrada por factores externos al estudiante). Según Alonso y Montero (1990, citados en León, 2002:106), "los cambios motivacionales operan en dos direcciones: por un lado tratar de aumentar la competencia percibida por los alumnos y por otro, reforzar sus experiencias de autonomía personal". A este respecto, Johnson y Johnson 
(1985, citados en León 2002:106) se suman a la creencia de que ese aumento de la competencia percibida por los alumnos y de la autonomía personal se generan y desarrollan en estructuras de aprendizaje de tipo cooperativo y escasamente o nada en estructuras de aprendizaje competitivas o individualistas. Del mismo modo, Lobato (1997:62) expone que:

La motivación a comprometerse nace del hecho de que el éxito de cada uno está ligado al éxito de los demás o de que la dificultad individual puede ser atenuada por la ayuda que se recibe del resto. Aunque puede ser una motivación extrínseca, no pasa de ser inicial o secundaria. Progresivamente la calidad de la relación entre los miembros, la ayuda recíproca, la estima mutua, el éxito, determinan una motivación intrínseca y convergente de todos los alumnos.

Con respecto a los incentivos que se pueden dar en el Aprendizaje Cooperativo destacan, por un lado, los incentivos externos (los externos directos y vicarios) y por otro, los incentivos internos autoproducidos o autogenerados. Los incentivos externos directos son los más indicados para estimular la participación en actividades que no despiertan por sí solas el interés del alumnado y que incluso llegaría a rechazar. Sin embargo, es conveniente administrar correctamente el uso de este tipo de incentivos, ya que algunos estudios demuestran algunas consecuencias negativas derivadas de su recurrencia frecuente, tal y como advierte Kohn (1990, citado en Arnold y Douglas, 2000:31): "en situaciones experimentales se ha demostrado que los sujetos manifestaban una reducción de la eficacia y el placer respecto a una tarea intrínsecamente interesante cuando se introducía una recompensa extrínseca" y en el mismo sentido Martínez (2001:243) advierte que "los refuerzos extrínsecos pueden reducir la motivación intrínseca o la motivación de logro".

Por su parte, los reforzadores vicarios son aquellos que recaen en el modelo, y proporcionan información al observador sobre los efectos beneficiosos o, por el contrario, perjudiciales de las conductas a imitar. En palabras de Rivas (2008:62):

[...] en lo relativo a los incentivos vicarios hay que señalar su peculiaridad en este tipo de aprendizaje, tal que, cuando se observa que la conducta de otra persona es recompensada, se propende a imitarla. Esto es, las consecuencias de la conducta del sujeto-modelo son de índole sustitutiva o vicaria para el aprendiz.

Finalmente, los incentivos internos, intrínsecos o autogenerados por el aprendiz, aunque más difíciles de lograr, poseen una notoria potencia en la regulación, dirección de la propia conducta y persistencia en el esfuerzo (Rivas, 2008:62). Rivière (1992:8) añade que el desarrollo de los mecanismos de autorrefuerzo y autoevaluación proporciona un sistema motivacional muy poderoso y que posibilita la realización, durante períodos largos de tiempo, de tareas difíciles o tediosas cuyas consecuencias externas pueden ser muy a largo plazo. La necesidad de desarrollar en los aprendices el incentivo autoproducido la establece Ruiz (2010:5) al afirmar que:

Las conductas autorreguladas tienden a mantenerse más que las reguladas desde instancias externas y, además, incrementan la percepción de capacidad personal de control sobre el medio. Por ello, los profesores deberían incrementar el tratar de desarrollar las capacidades de autoevaluación y autorrecompensa en sus alumnos y fomentar situaciones en que estos establecen por sí mismos los criterios para valorar sus rendimientos y actitudes. 
Por tanto, se concluye que la motivación, mediante las técnicas de Aprendizaje Cooperativo, contribuye positivamente en el desarrollo de la autonomía, fundamentalmente a través de los reforzadores vicarios así como los incentivos internos, intrínsecos o autoproducidos, puesto que contribuyen a direccionar la conducta o actitud de los educandos, estimulando una actitud más autónoma e independiente en su propio proceso de formación.

\subsection{Atención a los Estilos de Aprendizaje}

Un tercer aspecto a considerar en las técnicas de Aprendizaje Cooperativo para el desarrollo de la autonomía son los estilos de aprendizaje que podemos hallar en el aula de L2. Según López (1996:181) "uno de los planteamientos que el actual sistema educativo tiene como prioritario, es que se tenga en cuenta a la diversidad de intereses y capacidades de los alumnos desde el punto de vista docente y se obre en consecuencia". Cada sujeto adquiere de forma diferente los contenidos, dadas las diferencias entre sus ideas, pensamientos, experiencias así como sus estrategias cognitivas, que son en definitiva lo que les ayudará a dar significado, a adquirir esta información y a desarrollar su autonomía de un modo personal (Cuadrado et al., 2013:3).

De acuerdo con Rojas et al., (2006:53) "una primera aproximación al concepto de estilo de aprendizaje es la manera peculiar que tiene una persona para abordar una tarea de aprendizaje". A este respecto es interesante sumar la aportación de Marins de Andrade (2010:148) cuando expone que:

En cuanto a los estilos de aprendizaje, estos no son dicotómicos pues una persona puede ser más extrovertida que introvertida o igualmente visual o auditiva, así como puede aprender mejor individualmente y, sin embargo, disfrutar también con trabajos grupales. Unos estudiantes aprenden mejor con muestras orales de lengua y otros, en cambio, con muestras escritas; además, hay quienes necesitan manipular objetos y/o desarrollar una actividad física. Así, según la percepción sensorial los estilos pueden ser agrupados en cuatro áreas principales relativas a la percepción: visual, auditiva, cinestésica y táctil, además de las áreas de los sociológicos: grupal e individual. Por otro lado, hay diferencias de estilos según el tipo de personalidad (extravertidos/introvertidos, intuitivos/sensitivos, reflexivos/ sentimentales, estimadores/perceptores); según las diferencias biológicas (biorritmos, sostenimiento y la ubicación) y el temperamento (sanguíneo, melancólico, flemático y colérico).

El anterior extracto es especialmente relevante para nuestro objetivo, ya que nos proponemos, a través del Aprendizaje Cooperativo basado en la socialización y la interacción entre iguales, atender a todos los estilos de aprendizaje -sean sensoriales o de índole sociológica. Esta nueva forma de entender el trabajo en cooperación se apoya en la suma de los trabajos individuales de los miembros que conforman los grupos (la responsabilidad individual), con el doble objetivo de enriquecer los aprendizajes que se dan en las interacciones grupales y de atender a la diversidad de los individuos. Esta necesidad de atender a las características individuales de los estudiantes por parte de los docentes a través del Aprendizaje Cooperativo ha sido detectada por varios autores como Cuadrado et al., (2013:9) cuando afirman: 
[...] los estilos de aprendizaje son un referente a considerar por parte del profesorado para superar los hándicaps que de ellos se deriven en la búsqueda de una optimización del desarrollo de dichas competencias y la influencia que tienen en el uso adecuado de las TIC y en la utilización estratégica del aprendizaje colaborativo.

La atención a los distintos estilos de aprendizaje que fomenta el Aprendizaje Cooperativo permite la presencia de experiencias encaminadas al desarrollo de la autoconsciencia accediendo, de este modo, "a percepciones más profundas y ajustadas de sus respectivos puntos fuertes y débiles.” (García, 2005:114).

\section{Conclusiones}

Este artículo ha tenido como objetivo fortalecer los vínculos entre las técnicas de Aprendizaje Cooperativo y la autonomía del alumnado, dos campos que no siempre se ven interconectados. A lo largo del artículo, se han puesto de manifiesto los tres factores presentes en el Aprendizaje Cooperativo y que resultan fundamentales para desarrollar esta autonomía en el aula de L2 en todos los niveles: la reducción de la ansiedad, el aumento de la motivación, así como la atención a los estilos de aprendizaje. La implementación del Aprendizaje Cooperativo ayuda a los estudiantes a reducir los niveles de ansiedad a la hora de interactuar, además de mejorar su autoconcepto, la comprensión de sus puntos fuertes y débiles, sus estilos de aprendizaje y por ende, la motivación intrínseca y la actitud del educando hacia la propia tarea de aprendizaje.

Así pues, podría entenderse de esta reflexión teórica que, a través de las tareas cooperativas se motiva a los estudiantes, $\mathrm{y}$, a su vez, es la propia motivación la que hace al alumnado más autónomo en tanto que llega a alcanzar un nivel pleno de competencia y satisfacción por los objetivos logrados. En este efecto relacional que sucede en el Aprendizaje Cooperativo Arnold y Douglas (2000:32) señalan que:

[...] para estimular el crecimiento de la motivación intrínseca en el aula de idiomas: (1) ayudar a los alumnos a desarrollar la autonomía haciendo que aprendan a establecer metas personales y a utilizar estrategias de aprendizaje [...] (2) facilitar la participación de los alumnos a la hora de establecer algunos aspectos del programa y darles la oportunidad para realizar un aprendizaje en cooperación.

En definitiva, se trata de preparar al estudiante de manera aislada para integrar luego sus saberes en un grupo cooperativo potenciando sus capacidades personales, así como de implicarlo activamente en el proceso de aprendizaje que vive en aras de estimular su autonomía. Así, en la investigación llevada a cabo por León (2002:107) se afirma que:

[...] en determinados métodos de aprendizaje cooperativo, los alumnos deciden con el profesor aspectos relacionados con las variables del contexto (contenidos, organización de la actividad, tipo de tarea, evaluación...), lo que sin duda posibilita ese aumento de la autonomía en cuanto a la responsabilidad en el proceso de enseñanza-aprendizaje por parte de cada alumno. 
El desarrollo de una conducta autónoma por parte de los educandos no solo se va a ver estimulada por la participación en la toma de decisiones que repercuten en sus enseñanzas, sino que es la independencia misma con respecto al docente que promueve el Aprendizaje Cooperativo la que impulsará esta deseada conducta en los estudiantes (Otero, 2009:17).

\section{REFERENCIAS}

Ahmed, Z. y Zafar, S. (2010). "The effects of anxiety on cognitive processing in English language learning", in English Language Teaching, 3, 2: 199-209.

Aoki, N. (2000). "La afectividad y el papel de los profesores en el desarrollo de la autonomía del alumno", en J. Arnold, (ed.). La Dimensión Afectiva en el Aprendizaje de Idiomas. Cambridge: Cambridge University Press, 159-172.

Arnold, J. y Douglas, H. (2000). "Mapa del Terreno", en J. Arnold (ed.). La Dimensión Afectiva en el Aprendizaje de Idiomas. Cambridge: Cambridge University Press, 30-32.

Balçikanli C. (2010). "Learner autonomy in language learning: Student teachers' beliefs", in Australian Journal of Teacher Education, 35, 1: 90-103.

Barbero, E., Flores P., Jiménez, M., Martínez, J.A., Moreno, M. y Ruiz, E. (2001). "Towards Learner Autonomy", in GRETA. A Journal for Teachers of English, 9, 1: 28-48.

Basta, J. (2011). "The Role of the Communicative Approach and Cooperative Learning in Higher Education", in Facta Universitatis, 9, 2: 125-143.

Casal, S. (2005). Enseñanza del Inglés. Aplicaciones del Aprendizaje Cooperativo. Badajoz: Abecedario.

Coll, C. y Martín, E. (1999). "La evaluación del aprendizaje en el currículum escolar: Una perspectiva constructivista", en C. Coll, E. Martín, T. Mauri, M. Miras, J. Onrubia, Y. Solé y

A. Zabala. El Constructivismo en el Aula. Barcelona: Graó, 163-183. Competencias Clave para el Aprendizaje Permanente. Un Marco de Referencia Europeo (2007). Luxemburgo: Comunidades Europeas. Disponible en:

http://www.mecd.gob.es/dctm/ministerio/educacion/mecu/movilidad-europa/competenciasclave.pdf?documentId=0901e72b80685fb1 último acceso 31 de marzo, 2015.

Cuadrado, I., Fernández, I., Monroy, F.A. y Montaño, A. (2013). "Estilos de aprendizaje del alumnado de psicopedagogía y su implicación en el uso de las TIC y aprendizaje colaborativo", en RED. Revista de Educación a Distancia, 35: 1-19.

Deci, E.L, Vallerand, R.J, Pelletier, L.G., y Ryan, R. M. (1991). "Motivation and education: the self-determination perspective", in Educational Psychologist, 26, 3-4: 325-548.

Dörnyei, Z. 1994. "Motivation and motivating in the foreign language classroom", in The Modern Language Journal, 81/4: 482-493.

Denegri, M., Opazo, C. y Martínez, G. (2007). "Aprendizaje cooperativo y desarrollo del autoconcepto en estudiantes chilenos", en Revista de Pedagogía, 28, 81: 13-41.

García, M.E. y Cruz, M.L. (2013). "Factores motivacionales extrínsecos e intrínsecos en el aula de inglés: Análisis empírico", en Porta Linguarum, 19: 275-297

García Mata, J. (2005). "Aprendizaje en grupo y desarrollo de la autonomía individual en la formación de futuros profesores de lengua extranjera", en Porta Linguarum, 4: 109-120.

Gillies, R., y Ashman, A. F. (eds.) (2003). Co-operative Learning: the Social and Intellectual Outcomes of Learning in Groups. Londres: Routledge.

Horwitz, E. K., Horwitz, M. B. y Cope, J. (1986). "Foreign language classroom anxiety", in The Modern Language Journal, 70: 125-132. 
Johnson, D.W. (1993). "Impact of cooperative and individualistic learning on high ability students' achievement, self-esteem and social acceptance", in Journal of Social Psychology, 133, 6: 839-844.

Johnson, D., Johnson, R. y Holubec, E. (1994). Hacia un Aula Equitativa. Aprendizaje Cooperativo en la Educación Intercultural de Europa. Virginia: Association for Supervision and Curriculum Development.

Law, Y. (2008). "Effects of cooperative learning on second graders' learning from text", inEducational Psychology, 28: 567-582.

León del Barco, B. (2002). Elementos mediadores en la eficacia del aprendizaje cooperativo: entrenamiento en habilidades sociales y dinámicas de grupo. Tesis Doctoral. Departamento de Didáctica y Psicología. Universidad de Extremadura.

Lobato Fraile, C. (1997). "Hacia una comprensión del aprendizaje cooperativo", en Revista Psicodidáctica, 4: 59-76.

López García, J. (1996). "Los estilos de aprendizaje y los estilos de enseñanza. Un modelo de categorización de estilos de aprendizaje de los alumnos de enseñanza secundaria desde el punto de vista del profesor", en Anales de Psicología, 12, 2: 179-184.

MacIntyre, P.D. y Gardner, R.C. (1994). "The subtle effects of language anxiety on cognitive processing in the second language", in Language Learning, 44, 2: 283-305.

Marins de Andrade, P.R. (2010). "Estrategias de aprendizaje y desarrollo de la motivación: un estudio empírico con estudiantes de E/LE brasileños", en Porta Linguarum, 14: 141-160.

Martínez Agudo, J.D. (2001). "La activación y mantenimiento de la motivación durante el proceso de enseñanza-aprendizaje de una lengua extranjera", en Didáctica (lengua y literatura), 13: 238-261.

Moreno, R. y Martínez, R.J. (2007). “Aprendizaje autónomo. Desarrollo de una definición”, en Acta Comportamentalia, 15, 1: 51-62.

Otero, J.R. (2009). Qué-por qué-para qué-cómo. Aprendizaje Cooperativo. Propuesta para la Implantación de una Estructura de Cooperación en el Aula. Laboratorio de Innovación educativa. Madrid: Colegio Artica. Disponible en: http://www.jrotero.org/files/file/LABDGAC.pdf Consultado el 31 de marzo de 2015.

Picón, E.J. (2012). "Promoting learner autonomy through teacher-student partnership assessment in an American high school: a cycle of action research", in Profile. Issues in Teachers' Professional Development, 14, 2: 145-162.

Real Decreto 126/2014, de 28 de febrero por el que se establece el currículo básico de la Educación Primaria. BOE número 52, del 1 de marzo de 2014, disponible en:http://www. boe.es/boe/dias/2014/03/01/pdfs/BOE-A-2014-2222.pdf Consultado 31 de marzo, 2015. Recomendación del Parlamento Europeo y del Consejo de 18 de diciembre de 2006 sobre las competencias clave para el aprendizaje permanente (2006/962/CE). Diario Oficial de la Unión Europea L 394/10, disponible en: Recomendacion_Parlamento_Europeo_Consejo_Aprendizaje_permanente.pdf Consultado el 1 de abril, 2015.

Rivas Navarro, M. (2008). Procesos Cognitivos y Aprendizaje Significativo. Documento de Trabajo. Comunidad de Madrid: Consejería de Educación.

Rivière, A. (1992). La Teoría Social del Aprendizaje. Implicaciones Educativas. Madrid: Alianza.

Rojas, G., Salas, R. y Jiménez, C. (2006). "Estilos de aprendizaje y estilos de pensamiento entre estudiantes universitarios", en Estudios Pedagógicos, 22, 1: 49-75.

Rubio Alcalá, F. (2004). La Ansiedad en el Aprendizaje de Idiomas. Huelva: Servicio de Publicaciones de la Universidad de Huelva.

Ruiz Ahmed, Y. M. (2010). "Aprendizaje vicario: Implicaciones educativas en el aula”, en Revista Digital: Temas para la Educación, 10: 1-6. 
Ruiz de Zarobe, Y. (1997). "Aprendizaje autónomo en la adquisición de segundas lenguas: una experiencia en la universidad", en Didáctica, Lengua y Literatura, 9: 183-193.

Saravia, E. y Bernaus, M. (2008). "Motivación y actitudes para el aprendizaje de lenguas en dos colectivos de estudiantes universitarios: Futuros maestros de lengua extranjera y futuros enfermeros y fisioterapeutas", en Porta Linguarum, 13: 163-134.

Trujillo Sáez, F. (2002). "Aprendizaje cooperativo para la enseñanza de las lenguas", en Publicaciones, 32: 148-162.

Tsiplakides, I. (2009). "Helping students overcome foreign language speaking in the English classroom. Theoretical issues and practical recommendations", in International Education Studies. CCSE Journal, 2,4: 39-44.

Vera, M.M. (2009). “Aprendizaje cooperativo”, en Innovación y Ciencias, Revista Digital, 11: 1-11.

Vieira F., Barbosa, I., Paiva, M. y Fernandes, I.S. (2004). "Teacher education towards teacher (and learner) autonomy. What can be learnt from teacher development practices?", in T. Lamb y H. Reinders (eds.), Learner and Teacher Autonomy: Concepts, Realities and Responses. Amsterdam/Philadelphia: John Benjamins Publishing Company, 217-235.

Wang, P. (2011). "Constructivism and learner autonomy in foreign language teaching and learning: To what extent does theory inform practice", in Academy Publisher, 1,3: 273-277.

Xu, L. (2012). "The application of learner autonomy theory and model into ESP technology-assisted curriculum construction", in International Journal of English Linguistics, 2,5: 94-100.

Zoghi, M. y Nezhad, H. (2012). "Reflections on the what of learner autonomy", in International Journal of English Linguistics, 2, 3: 22-26. 